\title{
A NEW ACTION PRINCIPLE FOR WITTEN'S TOPOLOGICAL FIELD THEORY
}

\author{
R. Gianvittorio, A. Restuccia and J. Stephany \\ Universidad Simón Bolívar, Departamento de Física \\ Apartado postal 89000, Caracas 1080-A, Venezuela. \\ e-mail: ritagian@usb.ve, arestu@usb.ve,stephany@usb.ve
}

\begin{abstract}
In this letter a new gauge invariant, metric independent action is introduced from which Witten's Topological Quantum Field Theory may be obtained after gauge fixing using standard BRST techniques. In our model the BRST algebra of transformations, under which the effective action is invariant, close off-shell in distintion with what occurs in the one proposed by Labastida and Pernici. Our approach provides the geometrical principle for the quantum theory. We also compare our results with an alternative formulation presented by Baulieu and Singer.
\end{abstract}


The physical and mathematical properties of topological field theories have raised much interest in recent times (see for example Refs.[1-5]). In this letter we discuss an alternative approach for the construction of Witten's Topological Quantum Field Theory (TQFT) [1] using BRST techniques and starting from a new action principle different from the ones discussed in Refs.[2] and [3].

In Ref.[1] starting from a BRST-like supersymmetric principle Witten constructed a quantum effective action from which Donaldson invariants may be computed as correlation functions of adequatly choosen observables. In particular he showed, in the context of quantum field theory, the relation of Floer homology and Donaldson invariants.

Specifically the topological invariant path integral formula he obtained was

$$
Z\left(\gamma_{1}, \cdots, \gamma_{N}\right)=\int_{M} \mathcal{D} x \exp \left(\frac{-L^{\prime}}{e^{2}}\right) \prod_{i=1}^{N} \int_{\gamma_{i}} W_{k_{i}},
$$

where $\gamma_{i}$ is a $k_{i}$ dimensional homology cycle on $M$ and $W_{k_{i}}$ are structure functionals obtained from $W_{0}(P)=\frac{1}{2} \operatorname{Tr} \phi^{2}(P)$, $\phi$ being the spin zero field in his gauge fixed action. In (1) $L^{\prime}$ is the gauge fixed Lagrangean obtained from SUSY arguments ( see eq. (2.41) in $[1])$.

In the same paper [1] the problem of finding a manifestly generally covariant gauge theory whose BRST gauge fixing gives this gauge fixed Lagrangean was raised. Since then much work has been done to resolve this issue and important steps in the correct direction have been performed. The discussion on this point has been concentrated around two different actions proposed in Ref.[2] and Ref.[3] which have been shown to be related through the BRST formalism with Witten's TQFT.

The gauge invariant action proposed by Labastida and Pernici in [2] does indeed give rise after BRST gauge fixing to a TQFT which is identical to Witten's theory. The effective Lagrangean in [2], (see eq. (14) in [2]), is almost identical to $L^{\prime}$. It becomes identical when a sligth change in the gauge fixing conditions is performed (we discuss that point later on). Nevertheless since it is not independent of the metric on $M$, it do not represents the truly geometrical principle for the theory and the BRST algebra obtained in [2] by following the BV approach only closes on-shell.

In [3] the same problem was addressed with emphasis in exhibit a manifestly covariant gauge action, independent of the metric on $M$. The action proposed by Baulieu and Singer in [3], was the Pontryagin invariant

$$
\frac{1}{4} \int_{M} F \wedge F
$$

Its BRST gauge fixing leads to an effective Lagrangean $L$, (see eq.(2.13) in [1]) from which $L^{\prime}$ is obtained only after addition of a BRST invariant term of the same form as (2), that is

$$
L^{\prime}=L+\alpha F \wedge F .
$$

This last step which is completely valid in order to study the Donaldson invariants from a quantum field theory point of view, is an ad hoc modification of the BRST procedure and in fact does not resolve the problem we are considering. 
In what follows we introduce a new manifestly covariant gauge action, independent of the metric on M, whose BRST gauge fixing leads exactly to Witten's TQFT. The classical field equations we obtain are the same as in [2] but with the important difference mentioned above that they now arise from a variational principle of a metric independent gauge invariant action. Moreover the canonical formulation of the new action we are proposing also agrees exactly with the canonical formulation of the action in [2]. The effective lagrangean that we obtain is identical to Wittens $L^{\prime}$.

The action we propose is given by

$$
S=\frac{1}{4} \int \operatorname{Tr}(B+F) \wedge(B+F)=\frac{1}{4} \int d^{4} x \epsilon^{\mu \nu \sigma \rho}\left(F_{\mu \nu}^{a}+B_{\mu \nu}^{a}\right)\left(F_{\sigma \rho}^{a}+B_{\sigma \rho}^{a}\right) .
$$

where $F_{\mu \nu}^{a}$ is the curvature of the gauge connection $A_{\mu}^{a}$, with space-time index $\mu$ and group index $a ; B_{\mu \nu}^{a}$ is an independent antisymmetric field. It is important to mention that Horowitz in Ref.[7] introduces another topological gauge invariant action in terms of the same fields $B$ and $F(A)$, which nevertheless is BRST equivalent to take the Pontryagin invariant as was done by Baulieu and Singer in Ref.[3].

The action (3) is invariant under the infinitesimal non-abelian gauge transformations

$$
\begin{aligned}
& \delta A_{\mu}^{a}=\epsilon_{\mu}^{a}, \\
& \delta B_{\mu \nu}^{a}=-\mathcal{D}_{[\mu} \epsilon_{\nu]}^{a} .
\end{aligned}
$$

with $\epsilon_{\mu}^{a}$ the gauge parameters.

The field equations associated to (3) are

$$
F_{\mu \nu}^{a}+B_{\mu \nu}^{a}=0
$$

Evaluated over the classical solutions of the field equations, our action and the one proposed in Ref.[2] are zero . This is not the case before quantization for the action proposed in (2). In this case the solutions to the field equations are either selfdual or antiselfdual and evaluation of the action gives the corresponding Pontryagin index.

The gauge invariances of the action (3) allow to make the partial gauge fixation

$$
B_{\mu \nu}^{a+}=0,
$$

where

$$
\begin{aligned}
B_{\mu \nu}^{a( \pm)} & \equiv \frac{1}{2}\left(B_{\mu \nu}^{a} \pm \bar{B}_{\mu \nu}^{a}\right), \\
\bar{B}_{\mu \nu}^{a} & \equiv \frac{1}{2} \epsilon_{\mu \nu \sigma \rho} B^{\sigma \rho a} .
\end{aligned}
$$

The field equations then reduce to

$$
\begin{gathered}
F_{\mu \nu}^{a+}=0 \\
F_{\mu \nu}^{a-}+B_{\mu \nu}^{a-}=0,
\end{gathered}
$$


which are invariant under the remaining gauge freedom of (3), that is

$$
\begin{aligned}
& \delta A_{\mu}^{a}=\mathcal{D}_{\mu} \epsilon^{a}, \\
& \delta B_{\mu \nu}^{a}=0 .
\end{aligned}
$$

This field equations (7) are identical to the ones obtained in [2] by Labastida and Pernici. They have been obtained in our case from an action which is manifestly independent of the metric.

The action (3) may be also formulated in a canonical form. Then we obtain

$$
S=\int d^{4} x \dot{A}_{i}^{a} \epsilon^{i j k}\left(F_{j k}^{a}+B_{j k}^{a}\right)+A_{0}^{a} \mathcal{D}_{i}\left[\epsilon^{i j k}\left(F_{j k}^{a}+B_{j k}^{a}\right)\right]+B_{0 i}^{a} \epsilon^{i j k}\left(F_{j k}^{a}+B_{j k}^{a}\right)
$$

Here we recognize a theory with vanishing canonical Hamiltonian and with canonical conjugate momenta to $A_{i}^{a}$ given by

$$
\pi^{i a}=\epsilon^{i j k}\left(F_{j k}^{a}+B_{j k}^{a}\right)
$$

where $\epsilon^{i j k} \equiv \epsilon^{0 i j k}$, and with Lagrange multipliers $A_{0}^{a}$ and $B_{0 i}^{a}$ associated respectively to the following constraints

$$
\begin{aligned}
& \phi^{a}=\left(\mathcal{D}_{i} \pi^{i}\right)^{a}=\partial_{i} \pi^{i a}+\left(A_{i} \times \pi^{i}\right)^{a}=\partial_{i} \pi^{i a}+f^{a b c} A_{i}^{b} \pi^{i c}=0, \\
& \phi^{i a}=\pi^{i a}=0 .
\end{aligned}
$$

The algebra of the constraints is given by:

$$
\begin{aligned}
& \left\{\phi^{i a}(x), \phi^{j b}\left(x^{\prime}\right)\right\}=0, \\
& \left\{\phi^{a}(x), \phi^{b}\left(x^{\prime}\right)\right\}=f^{a b c} \phi^{c}(x) \delta^{3}\left(x-x^{\prime}\right), \\
& \left\{\phi^{a}(x), \phi^{i b}\left(x^{\prime}\right)\right\}=f^{a b c} \Phi^{i c}(x) \delta^{3}\left(x-x^{\prime}\right),
\end{aligned}
$$

which show that all constraints are first class. Nevertheless they are not linearly independent since they satisfy the following identity

$$
\left(\mathcal{D}_{i} \phi^{i}\right)^{a}=\phi^{a}
$$

and thus we are in front of a reducible theory $[8,9]$ with one level of reducibility. The corresponding the matrix of reducibility is given by:

$$
a=\left(\mathcal{D}_{i},-1\right)
$$

As we already mentioned one can show after several calculations that the canonical formulation of the action proposed in [2] exactly agrees with the results discussed above. It is interesting to note that the explicit dependence on the metric of the action of Labastida and Pernici [2] disappears in the canonical formulation, explaining from a different point of view why this theory is topological. The dependence on the metric of 
the associated functional integral may only arise from the gauge fixing functions but of course the functional integral is itself independent of the gauge fixing conditions.

It is also interesting to remark that the algebra of first class constraints (12) is the same for the theories proposed in Ref. [2] and Ref.[3] and also for the 4-dim BF topological field theory Ref.[10] whose action is given by

$$
\int_{M} B \wedge F .
$$

This action in principle is not related to Witten's TQFT. In facts the classical solutions of the BF theory are flat connections belonging to the different theta sectors. This space of classical solutions is thus different from the one associated to Witten's theory. The BF theory is usually classified [5] as a type Schwarz [4] topological theory in distinction to the Witten's type topological theories [1]. This distinction however does not seem to be relevant in this case. All the properties of both theories arises from the general BRST treatment of the corresponding metric independent gauge invariant actions. This include the property that the semiclassical aproximation is exact as we will discuss below.

To construct the BRST charge we follow Ref.[9] and introduce the minimal sector of the extended phase space expanded by the conjugate pairs:

$$
\left(A_{i}^{a}, \pi^{i a}\right) ;\left(C_{1}^{a}, \mu^{1 a}\right),\left(C_{1 i}^{a}, \mu^{1 i a}\right) ;\left(C_{11}^{a}, \mu^{11 a}\right)
$$

where $\left(A_{i}^{a}, \pi^{i a}\right)$ are the original canonical coordinates and $\left(C^{a}, \mu^{a}\right)$ are the canonical ghost and antighost associated to the constraints (11).

The off-shell nilpotent BRST charge is then given by:

$$
\begin{aligned}
\Omega=< & C_{1}^{a}\left(\mathcal{D}_{i} \pi^{i}\right)^{a}+C_{1 i}^{a} \pi^{i a}+C_{11}^{a}\left[\left(\mathcal{D}_{i} \mu^{1 i}\right)^{a}-\mu^{1 a}\right] \\
& -\frac{1}{2} C_{1}^{a} C_{1}^{b} f^{a b c} \mu^{1 c}-C_{1}^{a} C_{1 i}^{b} f^{a b c} \mu^{1 i c} \\
& -C_{11}^{a} C_{1}^{b} f^{a b c} \mu^{11 c}>
\end{aligned}
$$

where $\langle\cdots\rangle$ stands for integration on the space like continuous index. As we said, since the first class constraints of [2] are identical to (11) the BRST charge is the same. The Hamiltonian is zero, as it should be for a topological field theory.

We now define the non minimal sector of the extended phase space [9]. It contains extra ghosts, antighosts and Lagrange multipliers. First we introduce the C-fields

$$
C_{m}^{a}, C_{m i}^{a} ; \quad C_{m n}^{a}, C_{m n i}^{a} ; \quad m, n=1,2,3
$$

where at least one of the indices $m, n$ take the values 2 or 3 . In addition to these ghost, antighost and Lagrange multiplier fields we introduce the $\lambda$ and $\theta$ fields (Lagrange multipliers), also in the non minimal sector,

$$
\begin{aligned}
& \lambda_{1}^{0 a}, \lambda_{1 i}^{0 a} ; \lambda_{1 m}^{0 a} ; \quad m=1,2,3 \\
& \lambda_{11}^{1 a} ; \\
& \theta_{1}^{0 a}, \theta_{1 i}^{0 a} ; \theta_{1 m}^{0 a} ; \quad m=1,2,3 \\
& \theta_{11}^{1 a} .
\end{aligned}
$$


In this notation the 1 subscripts denote ghost associated to a gauge symmetry of the action, the 2 subscripts denote antighost associated to a gauge fixing condition in the effective action and the 3 subscripts denote Lagrange multipliers associated to a gauge fixing condition. The effective action is then given by:

$$
\begin{aligned}
\text { Seff }=\int_{t i}^{t f} d t\left[\pi^{i a} \dot{A}_{i}^{a}+\mu^{1 a} \dot{C}_{1}^{a}+\mu^{1 i a} \dot{C}_{1 i}^{a}+\mu^{11 a} \dot{C}_{11}^{a}+\right. \\
\left.\widehat{\delta}\left(\lambda_{1}^{0 a} \mu^{1 a}+\lambda_{1 i}^{0 a} \mu^{1 i a}+\lambda_{11}^{1 a} \mu^{11 a}\right)+L_{G F+F P}\right],
\end{aligned}
$$

where

$$
L_{G F+F P}=\widehat{\delta}\left(C_{2}^{a} \chi_{2}^{a}+C_{2 i}^{a} \chi_{2}^{i a}\right)+\widehat{\delta}\left(\sum_{m=1}^{3} C_{m 2}^{a} \chi_{m 2}^{a}\right)+\widehat{\delta}\left(\lambda_{12}^{0 a} \Lambda_{2}^{a}+\theta_{12}^{0 a} \Theta_{2}^{a}\right)
$$

is the sum of the generalizations of the Fadeev-Popov and gauge fixing terms. In Eq.(21) $\chi_{2}^{a}, \chi_{2}^{i a}$ are the primary gauge fixing functions associated to the constraints (11), while $\chi_{m 2}^{a}, \Lambda_{2}^{a}$ and $\Theta_{2}^{a}$ are gauge fixing functions which must fix the longitudinal part of fields of the non minimal sector. The BRST transformation for the canonical variables is given by

$$
\widehat{\delta} Z=(-1)^{\epsilon_{z}}\{Z, \Omega\}
$$

where $\epsilon_{z}$ is the grassmanian parity of $Z$. The BRST transformation of the variables of the non minimal sector are fixed imposing the closure of the charge as in Ref.[9]. After some simplifications we finally choose gauge fixing functions that may be written in a covariant form as

$$
\begin{aligned}
& \chi_{2}^{a}=\partial_{\mu} A^{\mu a}-\frac{\alpha}{2} C_{3}^{a}, \\
& \chi_{2}^{\mu \nu a}=\frac{1}{2} \epsilon^{\mu \nu \sigma \rho} B_{\sigma \rho}^{a}+B^{\mu \nu a} \\
& \chi_{12}^{a}=\mathcal{D}^{\mu} C_{1 \mu}^{a}+\frac{1}{2} C_{13}^{a} \times C_{11}^{a}+\frac{1}{2}\left(\left(C_{12} \times C_{1}\right) \times C_{11}\right)^{a},
\end{aligned}
$$

where $C_{1 \mu}^{a}=\left(-\lambda_{11}^{1 a}, C_{1 i}^{a}\right)$. After elimination of all conjugate momenta in the functional integral, the BRST transformation rules of all the remaining geometrical objects are covariant and take the form

$$
\begin{aligned}
& \widehat{\delta} A_{\mu}^{a}=-\mathcal{D}_{\mu} C_{1}^{a}+C_{1 \mu}^{a}, \\
& \widehat{\delta} C_{1}^{a}=C_{11}^{a}+\frac{1}{2}\left(C_{1} \times C_{1}\right)^{a}, \\
& \widehat{\delta} C_{1 \mu}^{a}=\mathcal{D}_{\mu} C_{11}^{a}+\left(C_{1} \times C_{1 \mu}\right)^{a}, \\
& \widehat{\delta} C_{11}^{a}=-\left(C_{11} \times C_{1}\right)^{a}, \\
& \widehat{\delta} C_{2}^{a}=C_{3}^{a}, \quad \widehat{\delta} C_{3}=0, \\
& \widehat{\delta} C_{2 \mu \nu}^{a}=C_{3 \mu \nu}^{a}, \quad \widehat{\delta} C_{3 \mu \nu}=0, \\
& \widehat{\delta} C_{12}^{a}=C_{13}^{a}, \quad \widehat{\delta} C_{13}=0 .
\end{aligned}
$$


The BRST invariant action, once we have eliminated $B_{\mu \nu}^{a}$, may be written as

$$
S=S_{0}+S_{1}+S_{2}
$$

where

$$
\begin{gathered}
S_{0}=<\frac{1}{4} F_{\mu \nu}^{a+} F^{\mu \nu a+}>, \\
S_{1}=<-C_{2}^{\mu \nu a} \mathcal{D}_{\mu} C_{1 \nu}^{a}+\frac{1}{8} C_{11}^{a}\left(C_{2}^{\mu \nu} \times C_{2 \mu \nu}\right)^{a}+\bar{C}_{13}^{a} \mathcal{D}_{\mu} C_{1}^{\mu a} \\
+C_{12}^{a}\left(C_{1}^{\mu} \times C_{1 \mu}\right)^{a}+C_{12}^{a} \mathcal{D}_{\mu} \mathcal{D}^{\mu} C_{11}^{a} \\
+\frac{1}{2} C_{11}^{a}\left(\bar{C}_{13} \times \bar{C}_{13}\right)^{a}-\frac{1}{2}\left(C_{12} \times C_{11}\right)^{a}\left(C_{12} \times C_{11}\right)^{a}>,
\end{gathered}
$$

with $\bar{C}_{13}^{a}=C_{13}^{a}+\left(C_{12} \times C_{1}\right)^{a}$, and finally

$$
S_{2}=<C_{3}^{a}\left(\partial_{\mu} A^{\mu a}-\frac{\alpha}{2} C_{3}^{a}\right)-C_{2}^{a} \partial_{\mu} \mathcal{D}^{\mu} C_{1}^{a}+C_{2}^{a} \partial_{\mu} C_{1}^{\mu a}>.
$$

It is interesting to note that the latest two terms in $S_{1}(27)$ which are of degree four and five in ghost and antighosts fields are due by the presence of terms of degree two and three in this field in the gauge fixing function $\chi_{12}^{a}$ in (23). This terms were not present in the gauge fixed action found in [2]. However since they arise from gauge fixing terms and the remaining term $\mathcal{D}^{\mu} C_{1 \mu}^{a}$ by itself is an admisible gauge fixing condition the gauge fixed action in [2] is equivalent to Witten's TQFT. In order to compare with Witten's formulation we perform the trivial change of variables:

$$
\begin{aligned}
& \psi_{\mu}^{a}=-i C_{1 \mu}^{a}, \\
& \lambda^{a}=i C_{11}^{a}, \\
& \eta^{a}=-\bar{C}_{13}^{a}, \\
& \phi^{a}=-2 i C_{12}^{a}, \\
& \chi_{\mu \nu}^{a}=-C_{2 \mu \nu}^{a},
\end{aligned}
$$

in $S_{1}(27)$. The resulting gauge fixed Lagrangean is then exactly $L^{\prime}$ [1].

We have introduced a new gauge invariant, metric independent, action which after BRST gauge fixing becomes identical to Witten's TQFT. The canonical formulation of the action we propose and of the one introduced by Labastida and Pernici are identical. The algebra of first class constraints of these actions is the same as the corresponding one for the theory proposed by Baulieu and Singer and also for the topological BF theory which certainly describes a non-equivalent topological theory. The BRST charges, however are different for these three theories.

The gauge action (3) is metric independent allowing a BRST topological treatment using the BFV $[[8,9]$ off-shell approach. Consequently the BRST transformation laws we obtain close off-shell, improving the results in Ref.[2].

The other interesting property we obtain is a new proof that in this theories the semiclassical approximation is exact. This property was proven by Witten [1] by explicit 
use of the particular structure of the action, being an anticonmutator involving the BRST charge. Instead we use the structure of the BRST charge (17), which is linear in the conjugate momenta. From this property it can be shown by inspection that the terms in Eq.(20) and Eq.(21) which do not involve the gauge fixing condition are

linear in the conjugate momenta. This implies that the gauge coupling constant $\frac{1}{e^{2}}$ of the effective action can be absorbed into the momenta by a change of variables in the functional integral. We are then left with the same effective action but in terms of a new gauge fixing condition. Since the functional integral is independent of the gauge fixing condition it implies the independence of any correlation function on the coupling constant. We may then take the limit $e \rightarrow 0$ as in Witten's argument and evaluate around the classical minima. This proof may be extended to all BF theories in any dimension [11].

\section{REFERENCES}

[1] E. Witten, Commun. Math. Phys. 117 (1988) 353.

[2] J. M. F. Labastida and M. Pernici, Phys. Lett. B212 (1988) 56.

[3] L. Baulieu and I. M. Singer, Nucl. Phys. (Proc. Suppl.) 5B (1988) 12.

Y. Igarashi, H. Imai, S. Kitakado and H. So, Phys. Lett. B227 (1989) 239.

C. Aragão and L. Baulieu, Phys. Lett. B275 (1992) 315.

[4] A. S. Schwarz, Commun. Math. Phys.67 (1979) 1.

[5] D. Birmingham, M.Blau, M. Rakowski and G. Thompson, Phys. Rep.209 (1991) 129.

[6] I. A. Batalin and G. Vilkovisky, Phys. Rev. D28 (1983) 2567.

[7] G. T. Horowitz, Commun. Math.Phys.125 (1989) 417.

[8] I. A. Batalin and E. Fradkin, Phys. Lett. B122 (1983) 157;Phys. Lett. 128 (1983) 307.

[9] M. I. Caicedo and A. Restuccia, Class. Quan. Grav 10 (1993) 833; Phys. Lett. B307 (1993) 77.

[10] M. Blau and G. Thompson, Ann.Phys. 205 (1991) 130.

[11] M. Caicedo, R. Gianvittorio, A. Restuccia and J. Stephany, in preparation. 